\title{
Early-access scheme picks controversial glioblastoma vaccine
}

A US-made cancer vaccine has become the first product to enter the UK's early-access pathway, a new scheme to fast-track patient access to investigational drugs for diseases with no adequate treatment. The UK's Early Access to Medicines Scheme (EAMS) was launched by the Medicines and Healthcare products Regulatory Agency (MHRA) in April 2014 (Nat. Biotechnol. 32, 403, 2014). In September, EAMS gave Bethesdabased Northwest Bio's DCVax-L dendritic cancer vaccine the first 'Promising Innovative Medicine' designation. But controversy has arisen over the choice of the vaccine and its US origins, and the program's running and future funding remain mired in uncertainty.

Criticisms that the UK scheme's first choice is a product from a US company is perhaps mistaking the aim of the process. This scheme is designed to accelerate approval of drugs for lifethreatening diseases from small and mediumsized companies, rather than to promote the UK biopharma industry. The selected DCVax-L is in phase 3 trials for glioblastoma multiforme, a brain cancer that is frequently fatal within two years. The vaccine takes a patient's own dendritic cells and loads them lysate prepared from the patient's own tumor tissue or cancer-related antigens. As well as offering a potential benefit for the most vulnerable patients, the EAMS process can also help small to medium-sized enterprises (SMEs), as the additional review of the data over and above the standard regulatory steps ratifies development programs by providing an early validation that the approach has potential, and facilitates earlier access to advice and support from regulatory authorities.

EAMS is a two-step process, says Daniel O'Connor, expert medical assessor, MHRA. As a first step, companies submit novel products they believe demonstrate significant benefit for patients in areas of high unmet need. The novel products must also offer an advantage over treatments already available in the UK. The assessment team makes a recommendation to the internal scientific consistency review group, which then advises the MHRA's director of licensing whether to grant the Promising Innovative Medicine designation. DCVax-L has successfully made it through this initial stage, but the second step, where the company's data will undergo a rigorous scientific assessment, is still to come.

Such scrutiny may allay concerns over dendritic cancer vaccines' mixed track record.
Prostate cancer vaccine Provenge (sipuleucel-T) made by Seattle-based Dendreon, has endured a rocky trajectory since its approval in 2010. The dendritic cancer vaccine has been under scrutiny for offering little benefit to patients (Nat. Biotechnol. 28, 882, 2010), and disappointing sales have ended in the company filing for bankruptcy on November 10.

Another concern voiced by Pamela Learmonth, director of public affairs and communications at the BioIndustry Association, is that no funding has yet been allocated. This pushes the costs of providing the treatment back to companies, and this could be a high hurdle for SMEs.

"The concept of the process is absolutely right-we need to get patients earlier access to medications in a controlled environment" says Paul Catchpole, value \& access director at the Association of the British Pharmaceutical Industry. However, it is important that there is a single, joined-up solution involving NICE [National Institutes for Clinical Excellence], the MHRA and the NHS [National Health Service], with all working together to achieve patient access.

Suzanne Elvidge Buxton, UK

\section{Around the world in a month}
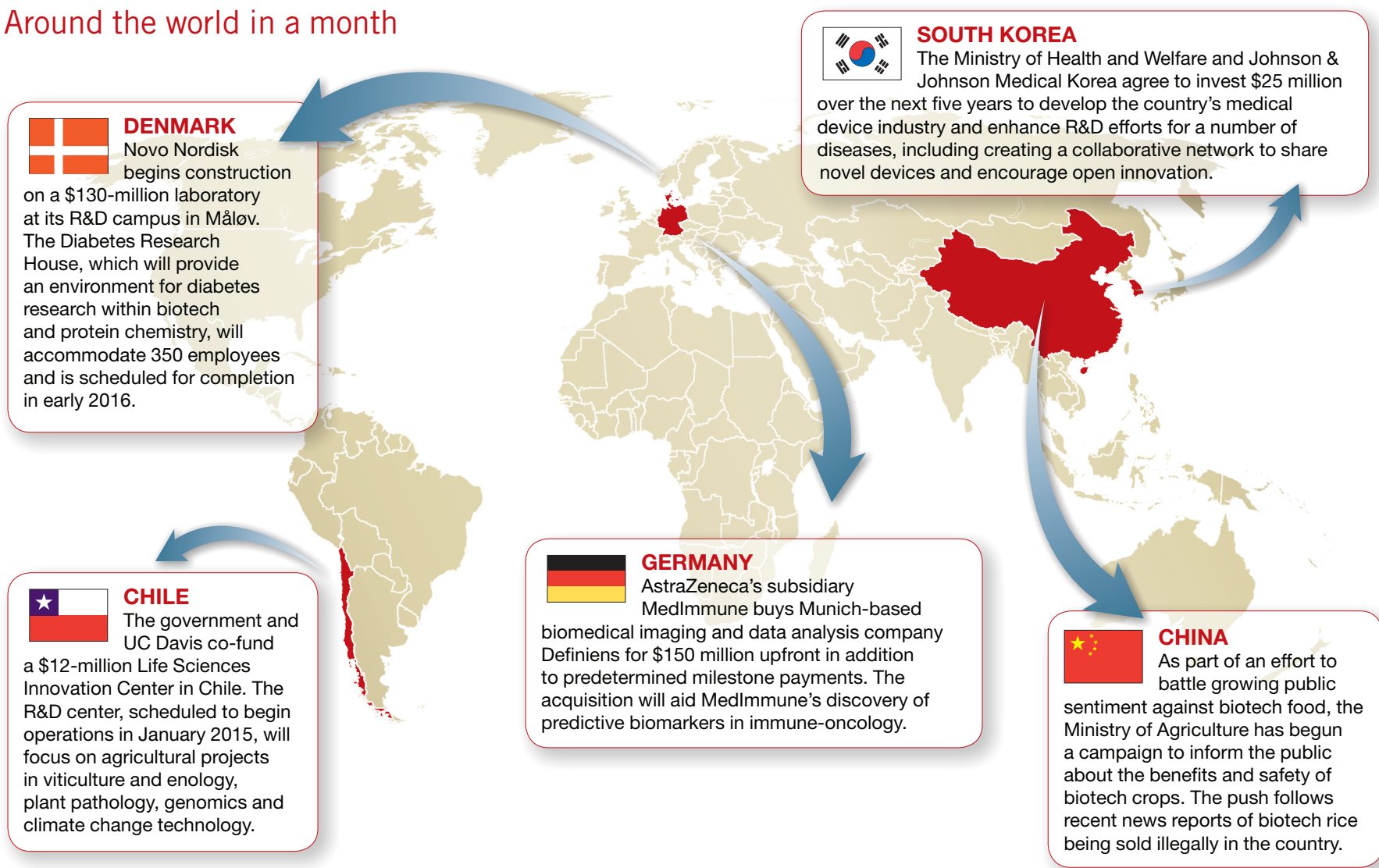